\title{
Conectores de extremidades de bambú para estructuras exploración de un sistema de incrustación en la pared interna del tallo
}

Connectors of bamboo extremities for structures exploration of an
increase system in the internal wall of the stem

\author{
YANN BARNET ${ }^{1}$ \\ FAOUZI JABRANE ${ }^{I}$
}

\section{RESUMEN}

Este estudio busca fomentar el uso del bambú en el sector de la construcción e innovar en el campo de las uniones desarrollando nuevas alternativas de conectores para la especie Guadua angustifolia. De forma totalmente distinta a las técnicas actuales, se explora las posibilidades de conectarse a la parte interna del tallo de bambú ejerciendo una fuerza mecánica en su pared, y evitar cortes especiales, perforaciones y el uso de cola o cemento. El resultado del estudio es una propuesta de patente de un dispositivo para conectar las extremidades de los bambúes con un sistema de expansión que penetra adentro del tallo y permite regular la dimensión de la pieza.

Palabras clave: construcción, bambú, uniones, conector

\begin{abstract}
This study seeks to encourage the use of bamboo in the construction sector and innovate in the field of unions by developing new alternatives for connectors for the Guadua angustifolia species. In a completely different way to the current techniques, we explore the possibilities of connecting to the internal part of the bamboo stem by exerting a mechanical force on its wall, and avoiding special cuts, perforations and the use of glue or cement. The result of the study is a patent proposal for a device to connect the extremities of the bamboos with an expansion system that penetrates inside the stem and allows to regulate the dimension of the piece.
\end{abstract}

Key words: construction, bamboo, unions, connector $\overline{1 \text { Universidad de }}$ San Martín de Porres, Perú

ybarnetc@usmp.pe

(C) Los autores. Este artículo es publicado por la Revista Campus de la Facultad de Ingeniería y Arquitectura de la Universidad de San Martín de Porres. Este artículo se distribuye en los términos de la Licencia Creative Commons Atribución No-comercial - Compartir-Igual 4.0 Internacional (https://creativecommons.org/licenses/ CC-BY), que permite el uso no comercial, distribución y reproducción en cualquier medio siempre que la obra original sea debidamente citada. Para uso comercial contactar a: revistacampus@usmp.pe. 


\section{Introducción}

\section{Ventajas del uso estructural del bambú en la construcción}

El bambú no es un árbol, sino una gramínea (familia Poaceae) que se puede asimilar a un pasto gigante. Está caracterizado por la subfamilia de los Bambusoideae que cuenta con más de 1600 especies alrededor del mundo (Vorontsova, y otros, 2016) concentradas, principalmente, en las zonas ecuatoriales y tropicales. La tribu Bambuseae, con unas 800 especies (Clark, 2006) es de particular interés para el uso en la construcción. En las regiones donde se encuentra existen usos milenarios en la arquitectura tradicional, sacando provecho de este recurso altamente renovable, reconocido como "eco-material" (Morán Ubedia, 2014) y con excelentes características físico-mecánicas.

Al ser un perfil natural muy ligero con una gran flexibilidad, el bambú ha probado ser un material idóneo para las zonas con riesgo sísmico. Tanto en Colombia, como en el Perú o en Ecuador, los recientes eventos sísmicos de gran magnitud evidenciaron el buen comportamiento estructural de las construcciones en bambú, sobre todo cuando las uniones son dúctiles (Kaminski, Trujillo, \& Lawrence, 2016).

En cada uno de esos países, se promulgaron normas de construcción afines que definen los métodos de diseño estructural y las buenas prácticas constructivas. Dentro de los bambúes "americanos", "sobresale la especie Guadua angustifolia, seleccionada como una de las 20 mejores del mundo por sus excelentes propiedades físico-mecánicas, su gran tamaño (20-30m) y por su com- probada utilización en la industria de la construcción” (Añazco, 2013).

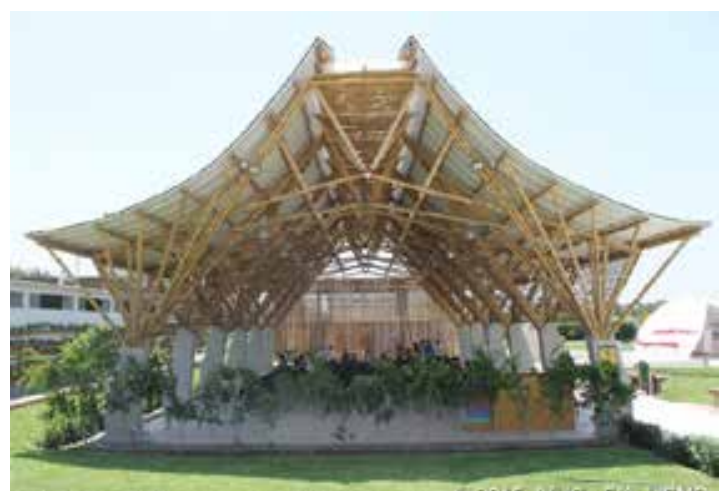

Figura1. Auditorio de bambú Voces por el Clima (Barnet \& Jabrane, 2017) - Lima, PERÚ Diseño: IVUC, USMP

\section{UNAS LIMITACIONES ACTUALES EN EL CAMPO DE LA CONSTRUC- CIÓN}

Pese a sus numerosas bondades, el bambú sufre de múltiples limitaciones para ser empleado, masivamente, como técnica constructiva. Además de una necesidad de difusión de buenas prácticas, y de caracterización físico mecánicas de numeras especies susceptibles de ser utilizadas a fines estructurales, Janssen resalta que una de las principales limitaciones actuales radica en las uniones: "En madera $o$ acero sabemos, después de un siglo de investigación, las propiedades de todos los tipos de sujetadores (clavos, tornillos, pernos, etc.) y con estos datos se pueden calcular los parámetros para cualquier unión. Pero en bambú, todavía tenemos que recorrer un largo camino." (Janssen, 2000).

En la actualidad, las guías y códigos elaborados en unos pocos países del mundo solo sustentan un uso bastante específico y limitado del bambú. Es importante recalcar que el bambú, a pesar de sus cualidades, es un material exigente cuyas características tienen que ser perfectamente controladas cuando está asumiendo un 
papel estructural de primer orden. Por su geometría cilíndrica y hueca, los tallos de bambú son sujetos al aplastamiento y ofrecen poca materia para los sujetadores. Además, por ser un perfil natural, ningún tallo es totalmente recto, la distancia entre los nudos varía, así como su sección (forma irregular, diámetros y espesores variables). Por todas esas razones, la estandarización de piezas es compleja y construir con bambú requiere de un saber hacer elaborado en el cual las uniones son un punto clave.

\section{OBJETIVOS DEL ESTUDIO}

Hoy en día los tiempos y el costo de la mano de obra toman siempre más relevancia en la construcción, por lo cual las técnicas necesitan ser cada vez más ágil. Para sacar ventajas de todas las bondades del bambú, el desafío es resolver unas limitaciones que lo segregan a un uso artesanal. El presente estudio está enmarcado en el proyecto de $\mathrm{I}+\mathrm{D}+\mathrm{i}$ "Diseńo de uniones y componentes industrializados para optimizar la construcción con bambú Guadua” cuyo principal objetivo es aportar soluciones técnicas concretas para aumentar la empleabilidad de la guadua sin pasar por procesos complejos y costosos de transformación. De forma específica, este estudio busca elaborar un nuevo método de conexión de las extremidades de los tallos de bambú, ya que resultan ser un punto crítico en el desarrollo de estructuras.

\section{Métodos y materiales}

El estudio se basa, principalmente, en el desarrollo y evaluación de prototipos en un proceso reiterativo de prueba-error que permite descartar métodos y acercarse a soluciones viables. De forma general, se siguió las siguientes etapas:

1. Vigilancia tecnológica para evaluar las técnicas actuales y elaborar nuevas estrategias de diseño y objetivos.

2. Diseño y fabricación de prototipos previsionales (uso de impresión 3D) con el fin de realizar pruebas volumétricas y ensayos funcionales.

3. Optimización de los prototipos y fabricación de modelos de prueba.

4. Pruebas funcionales con medición de tiempo.

5. Exploración del comportamiento estructural del conector a través ensayos de laboratorio. El principal interrogante de la solución planteada es su nivel de cohesión con el bambú. Por lo tanto, se realizaron principalmente ensayos a tracción.

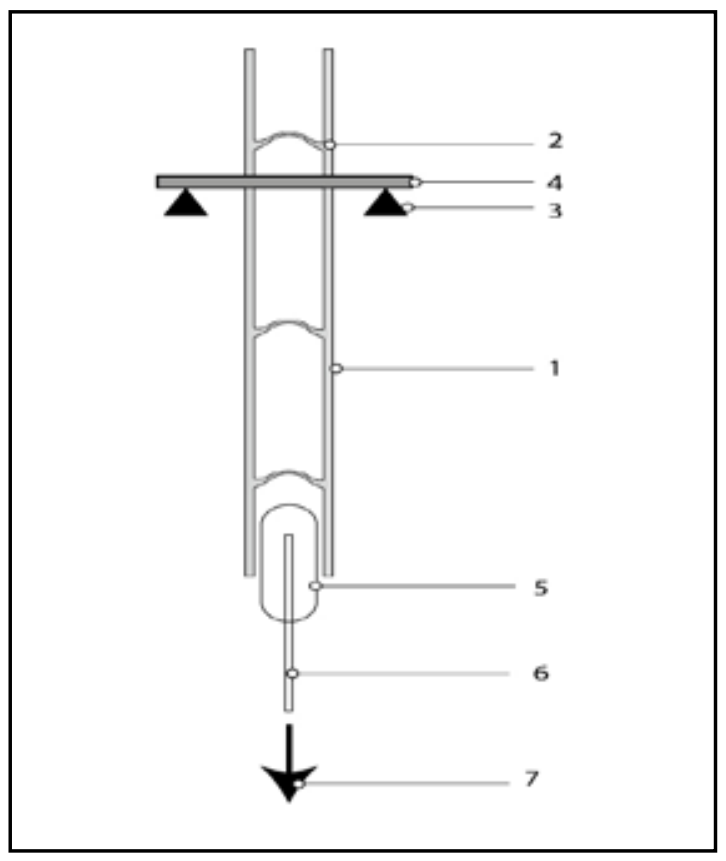

Figura 2. Esquema de los ensayos a tracción. 1- Guadua angustifolia, 2-nudo del bambú, 3-soporte fijo, 4- tubo de acero lleno de $25 \mathrm{~mm}$ de diámetro que perfora el tallo de bambú, 5-conector de extremidad ensayado, 6- varilla roscada galvanizada de $12 \mathrm{~mm}$ de diámetro, 7-fuerza aplicada

El bambú utilizado para los ensayos es de la especie Guadua angustifolia. Son tallos secos, pero no preservados. Se trató de escoger tallos maduros, pero sin tener 
garantías sobre su edad exacta. Diámetros exteriores utilizados: entre 90 y 120 m. m.

\section{Resultados y discusión}

\section{Vigilancia tecnológica para elaborar estrategias de diseńo}

En el mundo, el proceso actual para realizar uniones entre piezas de bambú es, principalmente, artesanal. Se realizan cortes especiales (boca de pescado, pico de flauta) que permiten el encaje entre la forma cilíndrica e irregular de los tallos de bambú con herramientas manuales (sierra, formón, martillo) o eléctricas como la caladora, el taladro con cierra de copa o la amoladora. Para fijar la unión se utilizan diversas técnicas, pero las principales son el uso de pernos pasantes, sogas, tarugos de madera o bambú.

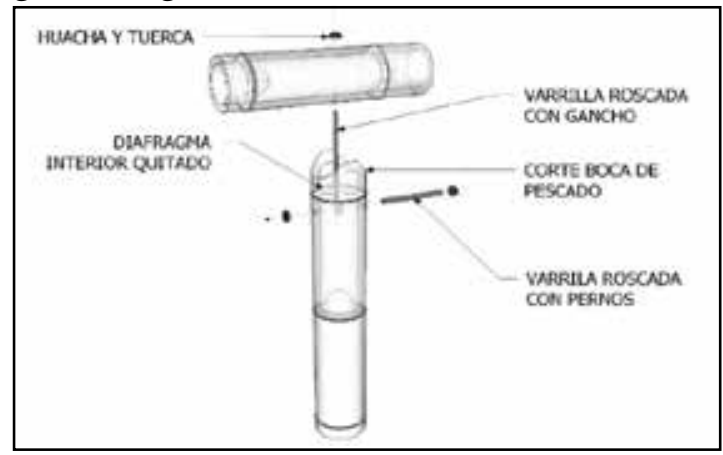

Figura 3. Unión perpendicular entre dos tallos de bambú con pernos pasantes. Corte Boca de pez. Sacado del Reglamento Nacional de Construcción del Perú (Ministerio de Vivienda, Urbanismo y Construcción, 2012)

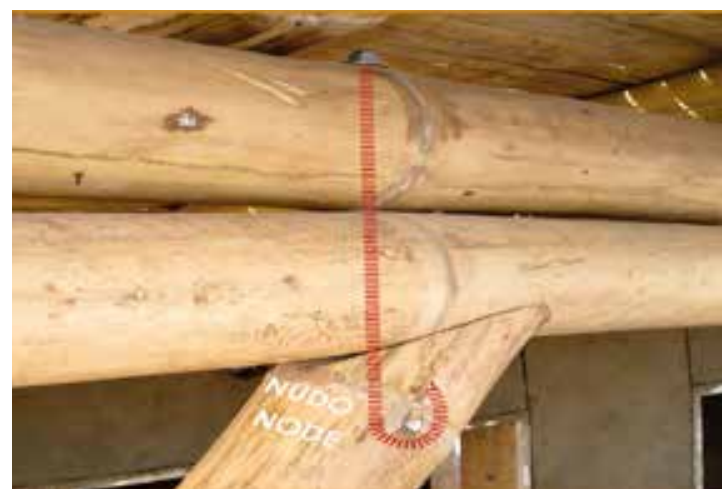

Figura 4. Conexión convencional con pico de flauta
Para las uniones que reciben esfuerzos importantes y son sujetas al aplastamiento del tallo existe la técnica del relleno con un mortero de los entrenudos expuestos. Se realiza perforando el bambú con sierra de copa y llenando manualmente con un embudo el interior del entrenudo. Obviamente, una vez efectuado este relleno, esas uniones no son desmontables.

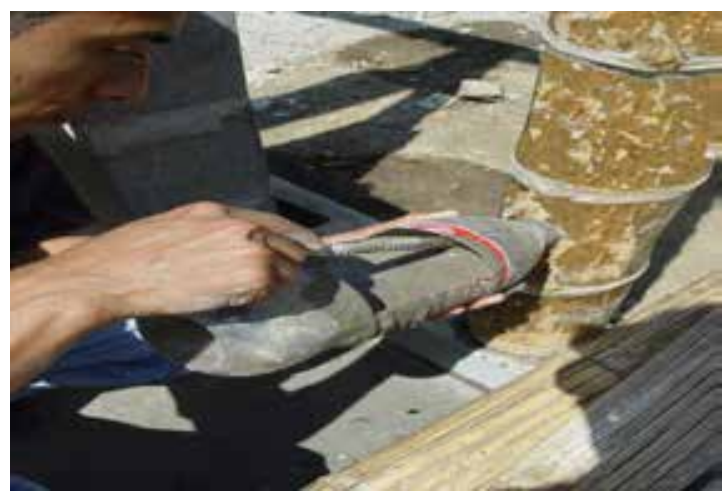

Figura 5. Relleno de canutos con cemento. Del Manual de construcción "Construir con bambú"

(Morán Ubidia, 2015)

Si bien esas técnicas son versátiles y requieren de conectores de bajo costo, demandan un saber-hacer especial y bastante tiempo. Para realizar una unión típica entre dos bambúes sobre el mismo plano, medimos que un especialista se demora unos 21 minutos: cinco minutos para escoger la caña con el buen dimensionamiento de entrenudos requerido, seis minutos para realizar el corte especial en boca de pescado o pico de flauta (según el ángulo deseado) y ocho minutos para asegurar los dos bambúes entre sí con un sistema de pernos pasantes. Según el costo horario de carpinteros especialistas, cada unión cuesta seis soles en mano de obra. Los materiales utilizados (varilla roscada con gancho, tuercas y arandelas, repuestos) cuestan un aproximado de tres soles. Por lo tanto, una unión tradicional cuesta un aproximado de nueve soles ( $1 \$=3.3$ soles). Por razones de resistencia, esas uniones tienen que ser ubicadas 
a proximidad de los nudos del bambú, lo que genera un desperdicio importante que se puede estimar a un mínimo de $25 \%$ de los tallos, representando un sobrecosto importante.

En varios partes del mundo, se han elaborado métodos alternativos para facilitar la conexión de los extremos de los tallos de bambú. A continuación, seleccionamos unos ejemplos puestos en práctica que consideramos representativos y de interés:

Conexión con piezas de madera tornada encoladas en la parte interna del bambú

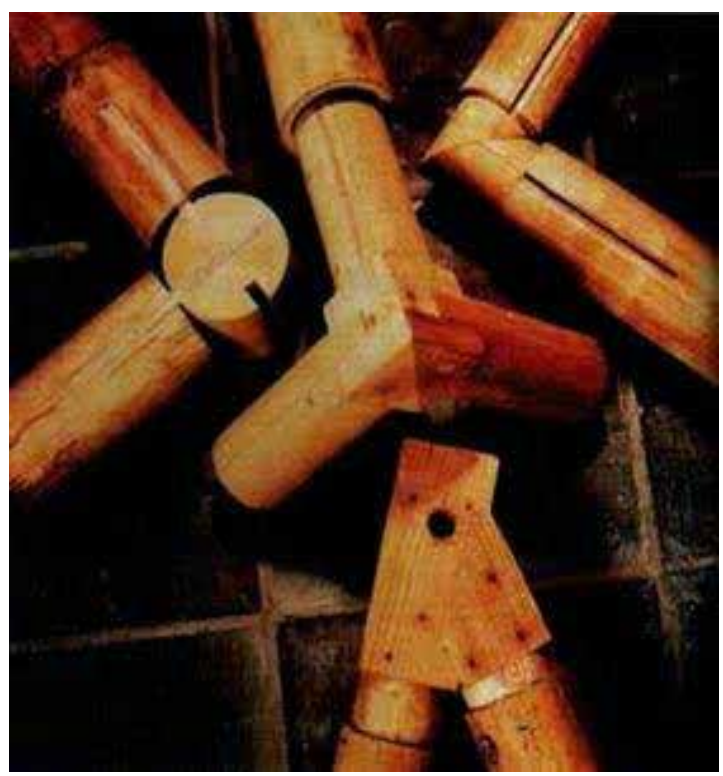

Figura 6. Conexión con piezas de madera de Villalobos (Arce-Villalobos, 1993)

Se realiza unos cortes longitudinales en la extremidad del tallo y se lija la parte interna del bambú donde se va a introducir un cilindro de madera tornada. La adhesión entre el bambú y la madera se realiza con cola, utilizando zunchos metálicos para asegurar la presión. Luego, se utilizan conectores y técnicas empleadas para la madera para realizar las conexiones.

\section{Conexión exterior con platinas metáli- cas y pernos pasantes}

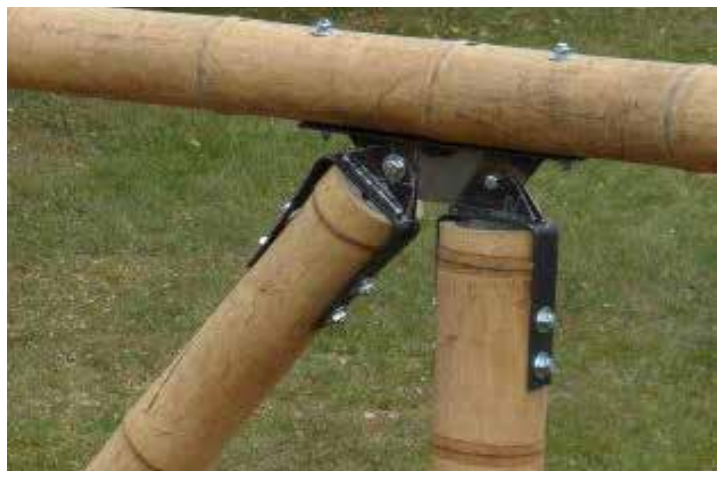

Figura 7. Conexión desarrollada por miembros de Fundeguadua- Colombia

Se emplean platinas metálicas fijadas a los tallos de bambú con pernos pasantes. Dichas platinas están diseñadas para ser fácilmente ensambladas (sistema de articulación).

\section{Conexión con pieza metálica embebi-} da en concreto

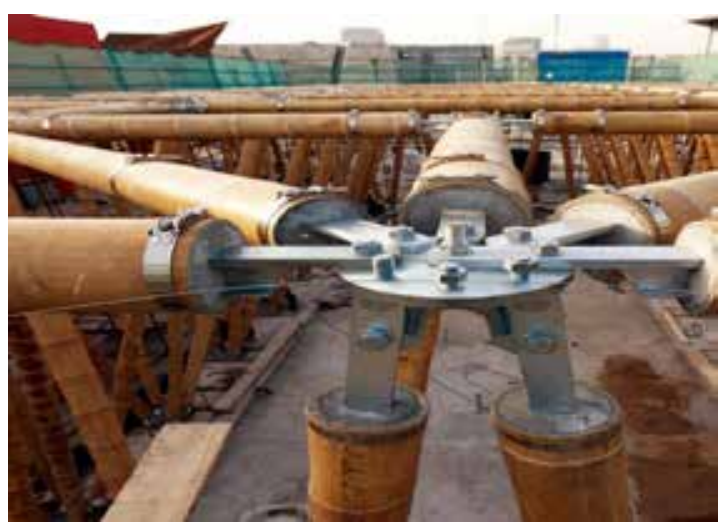

Figura 8. Conexión desarrollada por Markus Heinsdorff. (Heinsdorff, y otros, 2010)

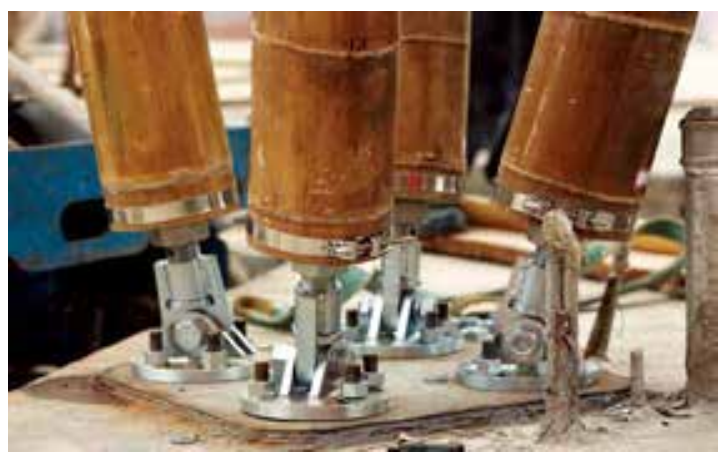

Figura 9. Conexión desarrollada por Markus Heinsdorff. (Heinsdorff, y otros, 2010). 
Se embebe una pieza metálica dentro del canuto con una mezcla de mortero. Puede ser platinas, perfiles o varillas en función al sistema de conexión deseado y la complejidad de los encuentros.

Conexión con pieza metálica embebida en concreto y extremidad cónica del tallo

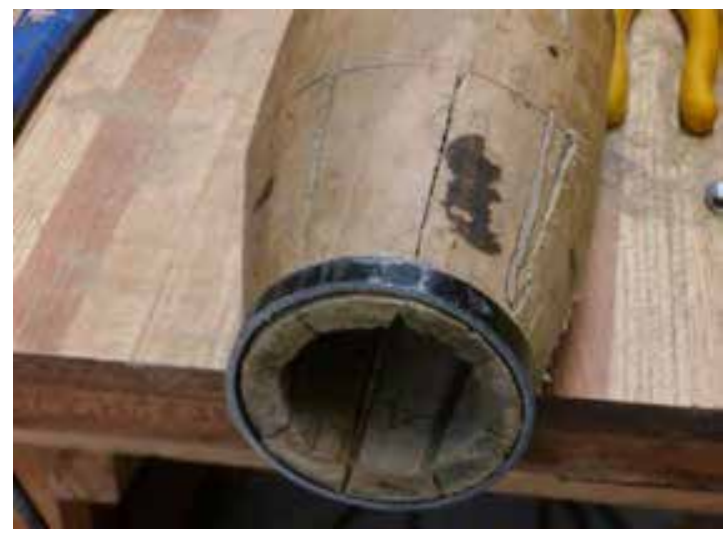

Figura 10. Proceso de fabricación de una extremidad cónica. Fotografía obtenida de la página www.bambutigre.it/strutture-inbambu

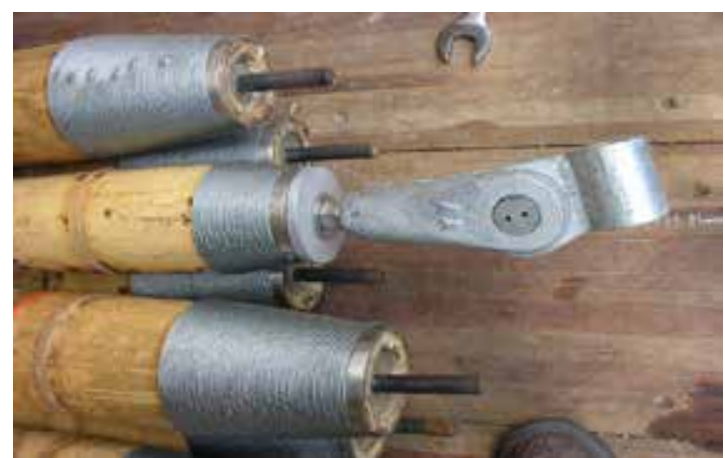

Figura 11. Conectores de Kool Bamboo, obtenida de su blog http://koolbamboo.blogspot.com

A la diferencia de la técnica anterior, se realizan cortes longitudinales en la extremidad del tallo con el propósito de crear una forma cónica asegurada por un anillo de metal. Eso permite aumentar la resistencia a la tracción de la unión, estandarizar el tamaño del diámetro final de la pieza de bambú y ofrecer un acabado interesante.
Como los ejemplos anteriores, existen también métodos que emplean piezas metálicas diseñadas y fabricadas de forma específica para responder a las necesidades de un diseño. Si bien pueden presentar un buen desempeño estructural y facilitar la realización de uniones en la obra, no son productos fabricados en serie y resultan muy onerosos para competir económicamente con el método tradicional.

\section{Estrategias de diseño del conector}

\section{- Funcionalidad}

Como varias propuestas actuales, se plantea que la extremidad del conector sea una varilla roscada, ya que permite acoplarse luego a toda una serie de conectores. Además, se propone que esta varilla pueda ser móvil ("enroscable") para regular ligeramente (mínimo $2 \mathrm{~cm}$ ) la longitud de la pieza, lo que dará una gran ventaja funcional versus los métodos existentes.

\section{- Sistema de montaje}

El conector pretende ser muy práctico y rápido de montar y eventualmente desmontar. Por lo tanto, tiene que prescindir de cualquier tipo de relleno de concreto o colas. La idea es utilizar un sistema de expansión que se adhiere a la parte interna del culmo de bambú y permita adaptarse a las irregularidades de las secciones de los bambúes. Como objetivo, se plantea diseñar un conector para bambúes con un diámetro exterior de $10 \mathrm{~cm}$ a $12 \mathrm{~cm}$.

\section{- Comportamiento estructural}

Es difícil comparar el desempeńo de los métodos de uniones porque varios no han sido ensayados en laboratorio. Sin embargo, existen unos datos: por ejemplo, las varillas embebidas dentro de una 
mezcla de concreto resisten un promedio de $1500 \mathrm{Kg}$ (Vavylousakis, 2016). Con bastante seguridad, podemos suponer que el método con extremidad cónica tendrá un mejor desempeño. El conector planteado tendría que ofrecer una resistencia similar a la propuesta con concreto pero más dúctil, lo que corresponde mejor al comportamiento del bambú.

\section{- Costos de fabricación}

Para ser competitivo con los otros métodos actuales, la estrategia es emplear piezas disponibles en el mercado con la menor cantidad posible de transformación, explorar métodos de fabricación digital y procesos industriales de fabricación masiva.

\section{Patente existente}

Hasta el momento, el único método que encontramos con un sistema de expansión es una patente china (文献, 2013), que describe un conector de extremidad de tallo de bambú compuesto de piezas móviles que giran sobre un eje y presionan la pared del bambú, empleando un cono en forma de cuńa situado en un mango redondo, el cual está encapsulado en un mango exterior atornillado al bambú.

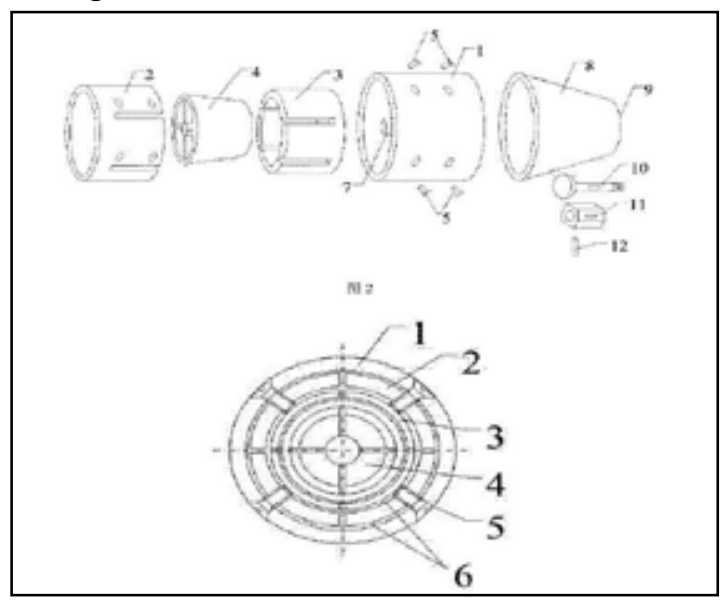

Figura 12. Esquemas de la patente $\mathrm{n}^{\circ} \mathrm{CN}$ 203284890 - El “2” representa el bambú. Las otras piezas son metálicas.
No encontramos pruebas de uso de esa invención y el dispositivo presenta varias desventajas técnicas: 1 .El tubo de sección fija en el cual tienen que encajar los bambúes limita el uso a tallos con un diámetro determinado. 2. La necesidad de realizar incisiones y perforaciones precisas en el bambú, lo que debilita la pieza y dificulta el trabajo. Se requiere pegamento. 3. No se puede regular la dimensión de la pieza. 4. Resulta complejo y costoso por ser compuesto de una docena de piezas diferentes.

\section{Diseño y evaluación funcional de prototipos}

Con el objetivo de simplificar el sistema y poder adaptarse a una cierta variación de diámetros de bambú se diseñó un conector basados en un taco "expansor" (2) que comprime tres cuñas (3) sobre la pared interna del bambú. Un disco de compresión (1) transmite esas cargas, y a la vez sirve de guía para el desplazamiento radial de las cuñas. Una varilla roscada (4) atraviesa el conector y tuercas permiten accionar el sistema de expansión. Un zuncho (5) evita la deformación y rotura del bambú.

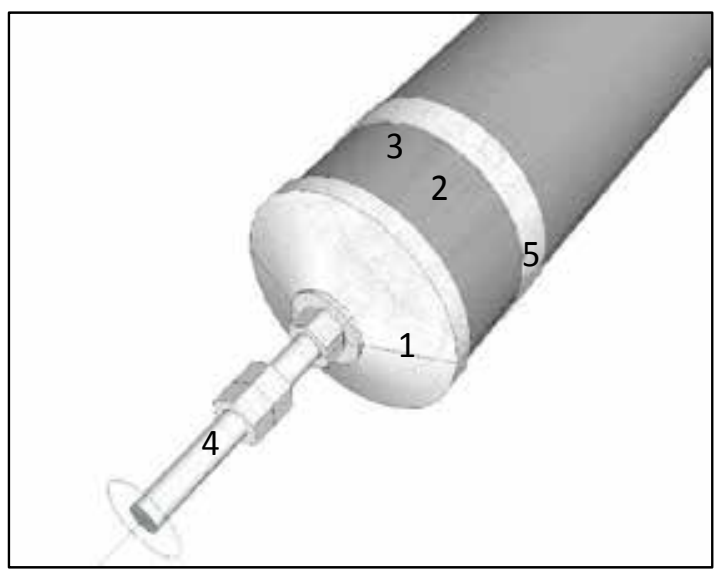

Figura 13. Diseño preliminar del conector

El primer prototipo se fabricó con pieza de madera. Con dos tipos de $\mathrm{cu}-$ 
ñas, unas con dientes y otras con recubrimiento de lija industrial.

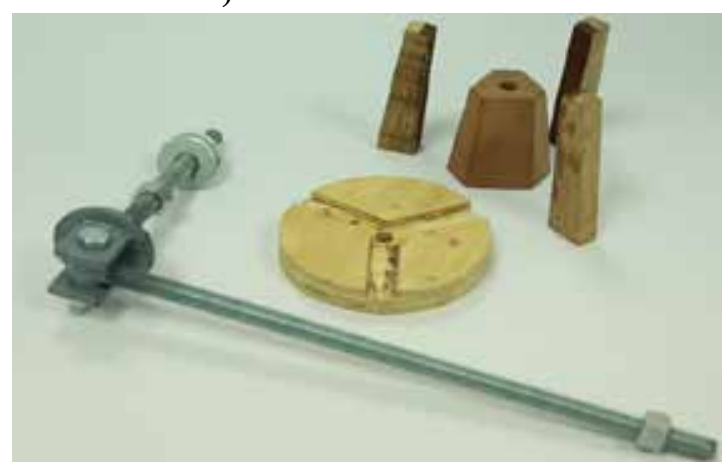

Figura 14. Prototipo I: madera, hecho a mano

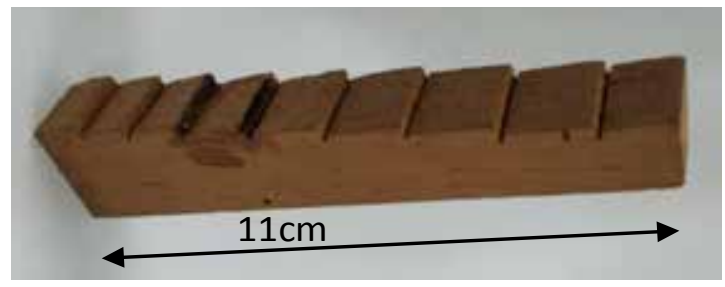

Figura 15. Prototipo de cuña de madera con dientes

Este primer prototipo demostró el buen funcionamiento de la geometría general, pero se detectó la necesidad de solidarizar las piezas entre sí para el montaje dentro del bambú y guiar el desplazamiento de las piezas. Por lo tanto, se añadió canales en forma de cola de milano que conectan las piezas en el siguiente prototipo que se fabricó con impresora 3D ya que la complejidad de los volúmenes hacía muy tediosa su fabricación en madera. Se pudo comprobar que las guías eran elementos muy útiles para una efectiva instalación en el bambú.

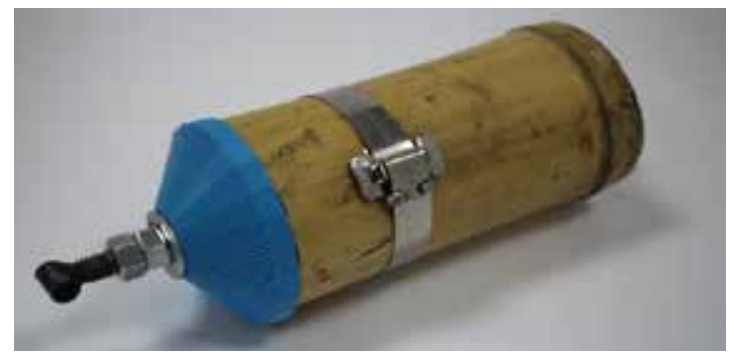

Figura 16. Prototipo II instalado: Plástico con impresora 3D, con guias tipo cola de milano mejoradas

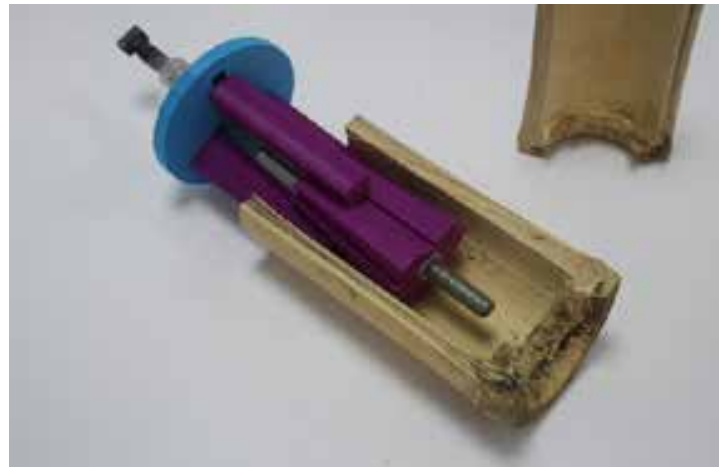

Figura 17. Prototipo II en bambú abierto

Posteriormente se han ido explorando opciones para aumentar el nivel de fricción entre el bambú y las piezas de plástico. En un prototipo III, se incorporó dientes en forma de pirámide capaz de incrustarse en la parte interna blanda del bambú.

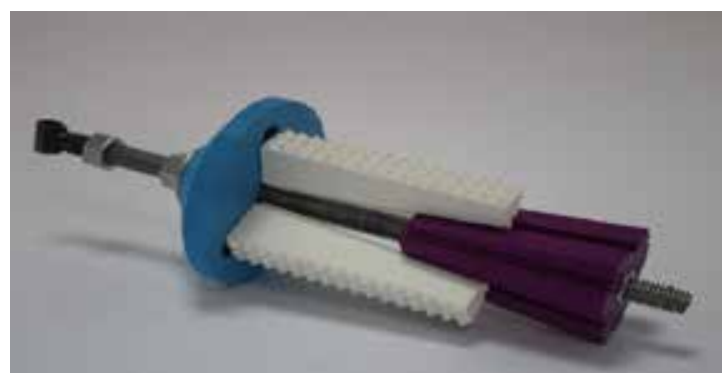

Figura 18. Prototipo III: Plástico con impresora $3 \mathrm{D}$, con cuñas con dientes

Este prototipo demostró un buen desempeño práctico en cuanto a su instalación ya que la colocación de la unión no supera cuatro minutos, incluyendo la colocación de un zuncho. Para la colocación de un segundo zuncho, se añade 2:30min.

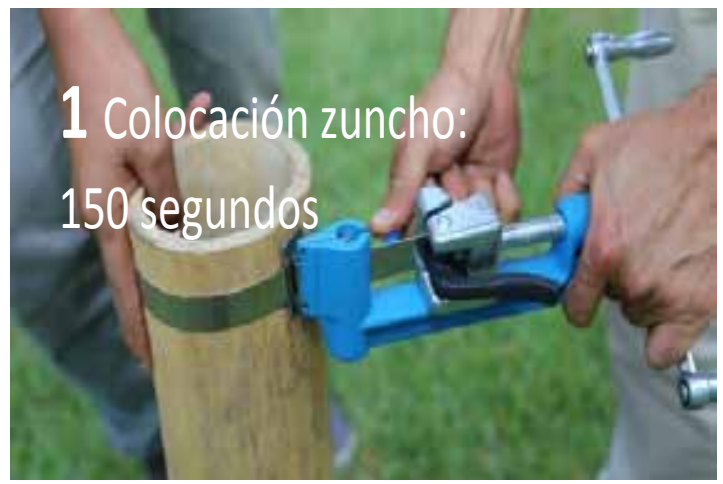



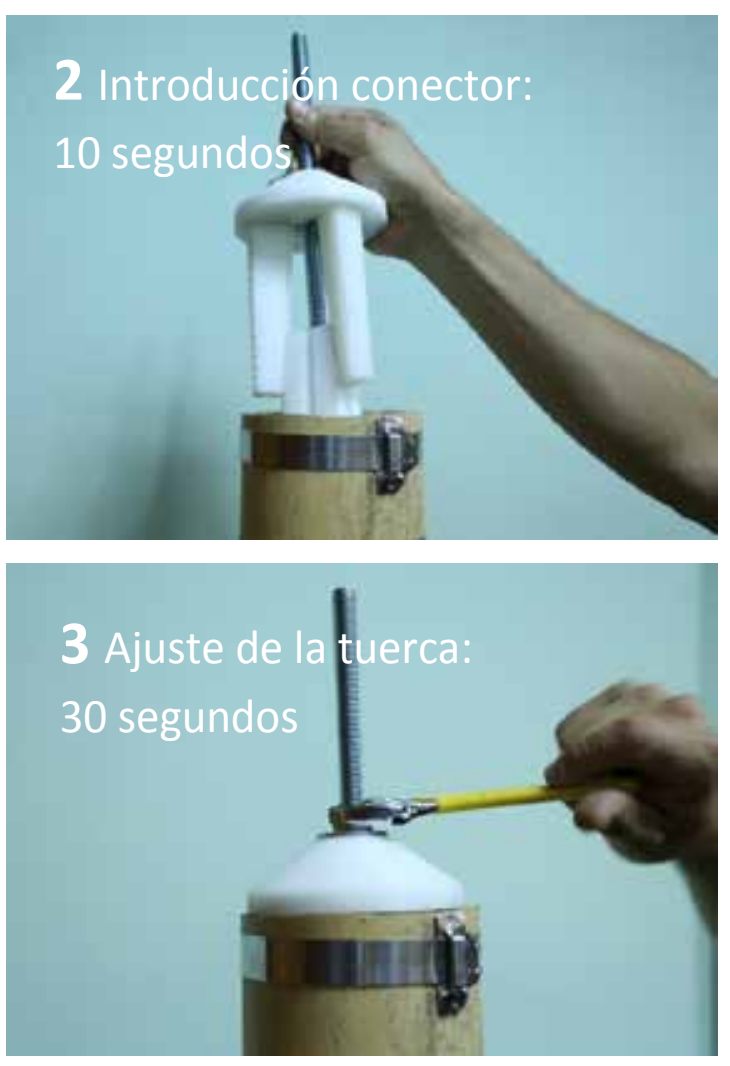

Figura 19. Proceso de montaje del prototipo de polietileno

En base a ese modelo, se fabricó un modelo de polietileno esculpido con fresadoras $\mathrm{CNC}$, que constituye una representación muy similar a lo que se conseguiría con un sistema de fabricación industrial en base a inyección en moldes, método que permitiría llegar a un costo de producción accesible. Para el caso específico del disco de compresión, se fabricaron también prototipos en madera densa con una fresadora $\mathrm{CNC}$, con el propósito de ofrecer alternativas de materiales y lenguaje arquitectónico.

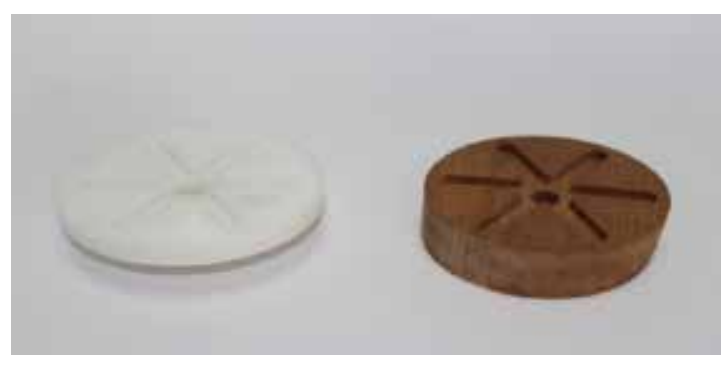

Figura 20. Discos de compresión en madera densa y polietileno

\section{Ensayos de prototipos bajo esfuerzos de tracción}

Por las características del conector desarrollado, la mayor incógnita es su comportamiento y resistencia cuando está sometido a tracción, ya que el esfuerzo de compresión resulta ser una transmisión sencilla de cargas de toda la superficie de la sección del tallo de bambú hacia el disco de compresión que a su vez transmite las cargas a la arandela, el perno y la varilla roscada. A cambio cuando está sometida a tracción el sistema "expansor" aplica un esfuerzo perpendicular a las fibras hacia el exterior del tallo, lo que agudiza la incrustación de los dientes de las cuñas dentro del bambú y provoca una deformación del tallo.

\section{Resultados del modelo con tres cuñas}

Los primeros ensayos presentaron un comportamiento no esperado a partir de cargas superiores a 200kg: a medida que se aumenta la fuerza de tracción, el "taco expansor" ha ido bajando y desplazando las cuñas hacia el exterior, provocando una deformación de la sección del bambú hacia una forma casi triangular, aun con la colocación de dos zunchos metálicos. Esta importante deformación dio la oportunidad al taco extensor chocar y empujar el disco de compresión como se nota en la siguiente foto. Lo que resultó satisfactorio fue la adherencia de las cuñas con el bambú, ya que no se desplazaron incluso después de la fisuración del bambú. 

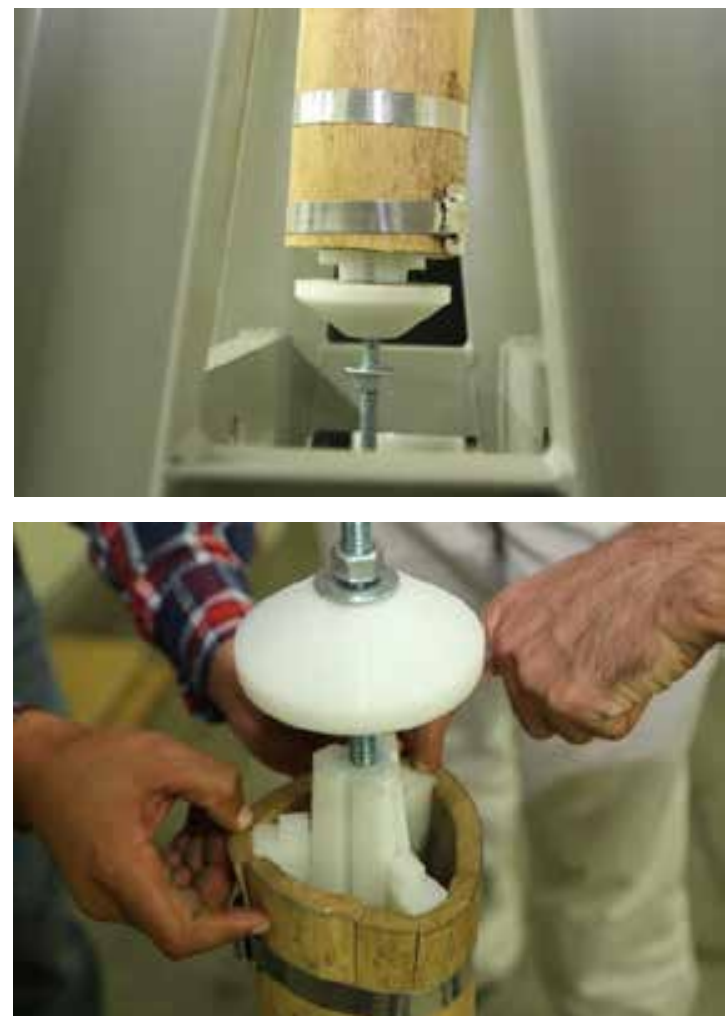

Figura 21. Desplazamiento con una fuerza aproximada de $400 \mathrm{~kg}$

\section{Resultados del modelo con seis cuñas}

Para evitar la deformación triangular evidenciada, se optó por fabricar nuevos prototipos con seis cuñas. Las pruebas realizadas demostraron un cambio radical del comportamiento de la unión: los desplazamientos empezaron alrededor de $400 \mathrm{~kg}$ de carga, y las primeras fallas (fisura ligera) del bambú surgieron superando una tracción de $650 \mathrm{~kg}$. Tales fisuras se produjeron primero en el lugar de la grampa de los zunchos. En los seis ensayos realizados, el colapso de la unión siempre ocurrió con una carga mayor a $1000 \mathrm{~kg}$. La carga más elevada de rotura final fue $1750 \mathrm{~kg}$, que coincidió con la rotura del zuncho más cercano de la extremidad del bambú. El desplazamiento a la rotura estuvo comprendido entre $7 \mathrm{~mm}$ y $20 \mathrm{~mm}$, lo que es una variación grande.

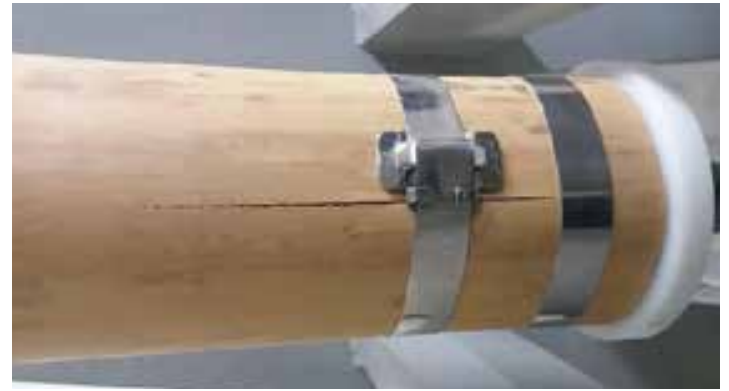

Figura 22. Primera falla: fisura en el lugar de la grampa del zuncho (carga $\geq 650 \mathrm{~kg}$ )

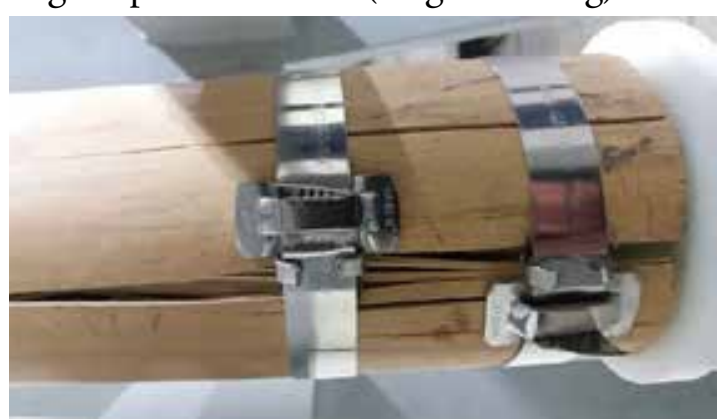

Figura 23. Rotura final: fisuras múltiples del bambú y apertura del zuncho en la zona de la grampa (carga $\geq 1000 \mathrm{~kg}$ )

Se probaron dos tipos de cuñas, unas con $18 \times 5$ hiladas de dientes, y otras con $3 \times 18$ hiladas de dientes. El promedio de resistencia de las probetas con más dientes es un poco superior, pero se necesitaría más ensayos para realmente sacar conclusiones detalladas al respecto.

Lo interesante es que, en ninguno de los ensayos, se produjo un deslizamiento de los dientes de plástico sobre el bambú, lo que comprueba que un sistema mecánico de incrustación en la parte interna de la pared de bambú es un método viable de conexión.

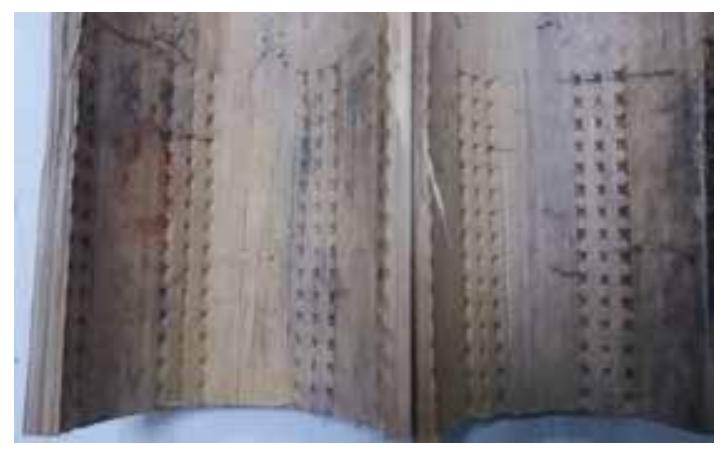




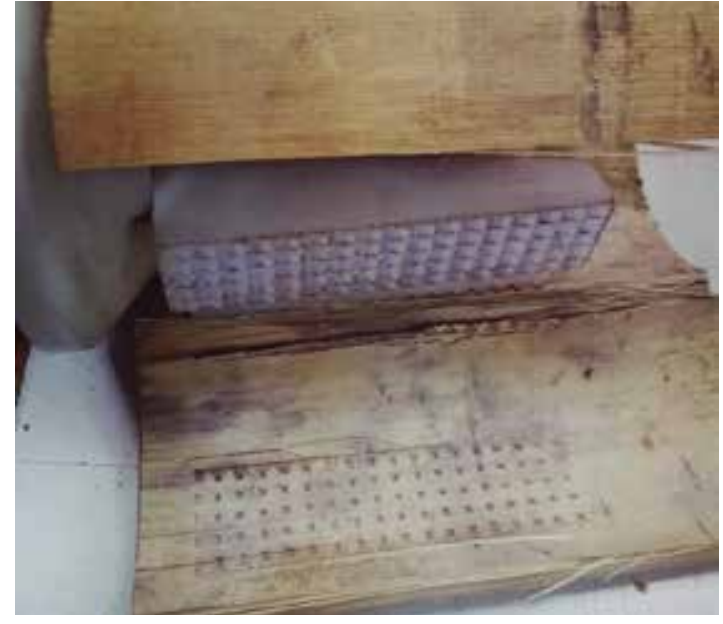

Figura 24. Incrustaciones en el interior del bambú después del ensayo a tracción. Arriba: cuñas con 18x3 hiladas de dientes Abajo: cuñas con $18 \times 5$ hiladas de dientes

\section{Nivel de adaptabilidad a secciones va- riables}

Con esos últimos prototipos, solo se consiguió que el conector se pueda adaptar a una variabilidad de $\mathbf{1 . 5} \mathbf{c m}$ de diámetro interior. Para tener más amplitud de uso, se podría plantear tener varios tamaños de "taco expansor", en función al diámetro del bambú que se quiere utilizar.

\section{Reflexión sobre los esfuerzos de corte}

En los casos de uniones que tienen que resistir un esfuerzo de corte perpendicular al tallo de bambú, este conector podría resultar débil porque la varilla roscada estaría sometida a un esfuerzo de flexión y podría doblarse. Ante este problema, se diseñó un disco de compresión cónico que ingrese al interior del bambú y pueda repartir este tipo de carga hacia el tallo. Sería necesario realizar ensayos al corte para evaluar el comportamiento estructural de la unión con la solución planteada.

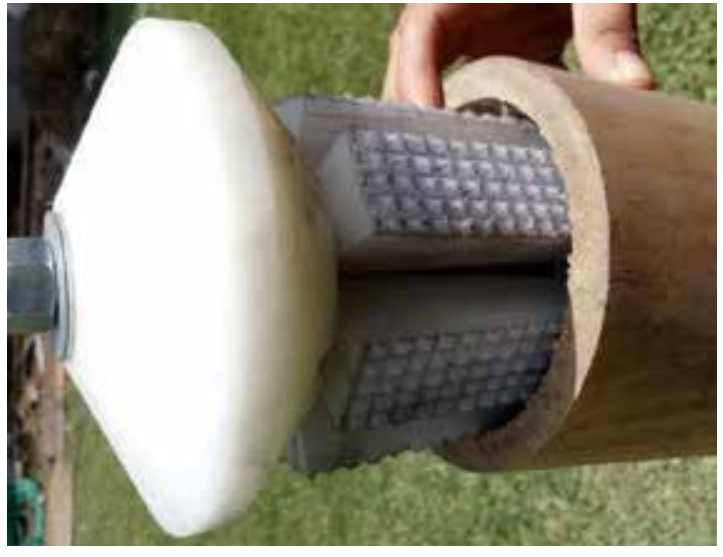

Figura 25. Prototipo de conector con disco de compresión cónico.

\section{Conclusiones}

El estudio ha permitido comprobar que es viable utilizar un método de incrustación mecánica en la parte interna de la pared de bambú, ya que no se presentó ningún deslizamiento, aun con fuerzas de tracción superior a $1500 \mathrm{~kg}$. Los ensayos exploratorios del conector con sistema de expansión han demostrado un comportamiento estructural alentador. Trabaja de forma idónea a compresión paralela al tallo de bambú, pero presenta una debilidad en cuanto a los desplazamientos relativamente altos si el conector está sometido a una fuerza de tracción alta. Por otro lado, se tendría que estudiar cuál es su comportamiento para el corte perpendicular al tallo de bambú.

El conector desarrollado presenta las siguientes ventajas técnicas:

- Es de muy fácil uso y no requiere de cortes especiales del bambú. Permite a una persona no experta realizar uniones entre bambúes con herramientas manuales de uso simple: llave, zunchadora manual.

- No requiere de un nudo a proximidad para realizar la unión, lo que per- 
mite disminuir el tiempo de elección del tallo y reducir los desperdicios de bambú.

- No requiere del uso de mortero ya que su sistema de expansión con los zunchos evita el aplastamiento del tallo. Eso evita trabajo, tiempos de secado del mortero y reduce el peso de la unión.

- Una vez asegurado el conector con el bambú, el dispositivo permite regular la dimensión de la pieza con una llave. Ofrece un juego de 2 a $3 \mathrm{~cm}$ que resulta muy importante para adaptarse a las irregularidades dimensionales características de los tallos de bambú.

- La unión puede ser desarmada de forma muy sencilla y rápida con una llave.

Esas ventajas han permitido presentar un proyecto de patente en el Perú (publicado en la Gaceta Electrónica de Propiedad Industrial de INDECOPI con el número de expediente 1321-2017).

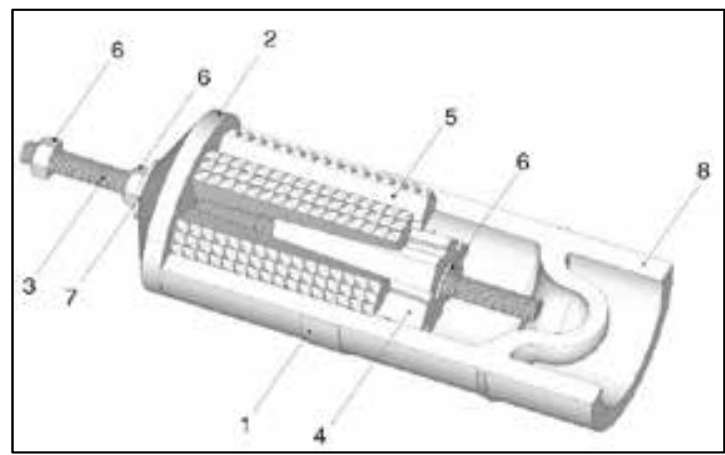

Figura 26. Una de las figuras del proyecto de patente donde se muestra una vista isométrica del dispositivo conector colocado en el tallo de bambú (8) parcialmente abierto para visualizar la interacción entre la invención y la pared interna del tallo de bambú (8). Se aprecia cómo las cuñas de fijación (5) se incrustan en la pared interna del tallo del bambú y se ven la varilla roscada (3) con las tuercas (6), arandela (7), el disco de compresión (2) y el taco extensor (4).

En importante recalcar que, para ser competitivo, este conector tiene que producirse en serie con el proceso de inyección de plástico. Sería interesante evaluar la posibilidad de utilizar plástico reciclado.

\section{Reconocimientos}

Se agradece al Ing. Industrial Rafael Figueroa, por sus aportes en el diseño y fabricación de prototipos, y al Arq. Víctor Barraza, por su apoyo en la fabricación y ensayos de prototipos.

\section{REFERENCIAS}

Añazco, M. (2013). Estudio de vulnerabilidad del bambú (Guadua angustifolia) al cambio climático en la costa del Ecuador y norte Perú. Quito: INBAR.

Arce-Villalobos, O. A. (1993). Fundamentals of the design of bamboo structures. Eindhoven: technische Universiteit Eindhoven.
Barnet, Y., \& Jabrane, F. (2017). Diseño de proyectos con bambú en Lima como estrategia de difusión de un método constructivo alternativo y sostenible. Campus $N^{\circ} 23,85-104$.

Clark, L. (2006). Bamboo biodiversity. Obtenido de http://www.eeob. iastate.edu/research/bamboo/index.html 
Heinsdorff, M., Baumert, C., Baur, M., Garrecht, H., Kahn-Ackermann, M., Knapp, G., Von Vegesack, A. (2010). Design with Nature The bamboo architecture. Munich: Hirmer.

Janssen, J. J. (2000). Designing and Building with Bamboo,. Beijing, Republica Polpualr de China: International Network for Bamboo and Rattan.

Kaminski, S., Trujillo, D. J., \& Lawrence, A. (2016). Structural use of bamboo: Part 1: Introduction to bamboo. The Structural Engineer, 40-43.

Ministerio de Vivienda, Urbanismo y Construcción. (2012). E100 Bambú. Reglamento Nacional de Edificaciones. Lima, Perú.
Morán, J. (2014). De los "Mate- riales convencionales" a los "Ecomateriales". Primer congreso Internacional Innovaciones con bambú. Guayaquil.

Morán, J. (2015). Construir con bambú "caña de Guayaquil". Lima, Perú: Red Internacional de Bambú y Ratán.

Vavylousakis, I. (2016). Research into Bamboo connections for seismic loadings. Coventry: Coventry University.

Vorontsova, M., Clark, L., Dransfield, J., Govaerts, R., Wilkinson, T., \& Baker, W. (2016). World Atlas of Bamboos and Rattans. Beijing: INBAR.

文献, 民. 杨. (2013). Patente $n^{\circ} C N$ 203284890. China. 
